\title{
Synthesis of Nanocrystalline CdS by SILAR and Their Characterization
}

\author{
Partha Protim Chandra, ${ }^{1}$ Ayan Mukherjee, ${ }^{2}$ and P. Mitra ${ }^{2}$ \\ ${ }^{1}$ Department of Physics, Banwarilal Bhalotia College, Asansol, Burdwan, West Bengal 713303, India \\ ${ }^{2}$ Department of Physics, The University of Burdwan, Golapbag, Burdwan, West Bengal 713104, India
}

Correspondence should be addressed to P. Mitra; mitrapartha1@rediffmail.com

Received 7 January 2014; Accepted 15 May 2014; Published 17 June 2014

Academic Editor: Zainuriah Hassan

Copyright (C) 2014 Partha Protim Chandra et al. This is an open access article distributed under the Creative Commons Attribution License, which permits unrestricted use, distribution, and reproduction in any medium, provided the original work is properly cited.

\begin{abstract}
A simple and cost effective chemical technique has been utilized to prepare cadmium sulphide (CdS) nanoparticles at room temperature. The sample is characterized with XRD (X-ray diffractometer), SEM (scanning electron microscope), TEM (transmission electron microscope), FTIR (Fourier transform infrared), EDX (energy dispersive X-rays), and UV-VIS (ultraviolet visible) spectrophotometer. The particle size estimated using X-ray line broadening method is $\sim 21.5 \mathrm{~nm}$. While particle size estimation, both instrumental and strain broadening was taken into account. The lattice strain was evaluated using WilliamsonHall equation. SEM illustrates formation of submicron size crystallites and TEM image gives a particle size of $\sim 23.5 \mathrm{~nm}$. The characteristic stretching vibration frequency of CdS was observed in the absorption band in FTIR spectrum. Optical absorption study exhibits a band gap energy value of about $2.44 \mathrm{eV}$.
\end{abstract}

\section{Introduction}

Semiconductor nanoparticles are an important class of materials with numerous applications in the fabrication of optoelectronic devices, photonic transducers, and photoluminescent tags for biological studies [1]. Nanometer-sized semiconductors exhibit structural, electronic, optical, luminescence, and photoconducting properties very different from their bulk properties [2,3]. Large-scale synthesis of semiconductor nanoparticles such as solid powder is critically important not only for the study of their physical properties but also for their industrial applications in the areas of catalysis, photocatalysis, and microelectronics. Cadmium sulphide is an important semiconductor and has many optoelectronic applications including solar cells, photodiodes, light emitting diodes, nonlinear optics, heterogeneous photocatalysis, high-density magnetic information storage, and many others in semiconductor industries $[4,5]$. The characteristic absorption of light for $\mathrm{CdS}$ is in the visible range with a bulk band gap of $\sim 2.4 \mathrm{eV}$ at room temperature [5]. Many of these nanoparticles can be prepared using simple wet-chemical methods of synthesis that allow one to manipulate the electronic properties of the particles [6-9]. In powder form, CdS has been synthesized using hydrothermal/solvothermal methods, thermal decomposition [10-12], single-molecule precursors approach [13], and chemical precipitation technique with or without capping agent [4].

In the presented work, a chemical dipping technique has been utilized to prepare nanopowders of CdS at room temperature. The technique involves successive dipping of a precleaned substrate in separately placed cationic and anionic precursor. Between every immersion it is rinsed in ion exchanged water. The technique, often called SILAR (successive ionic layer adsorption and reaction), is generally reported for preparation of thin films of cadmium sulphide [14].

Normally under optimized deposition conditions, SILAR produces adherent thin films. The deposition parameters for getting adherent thin films include concentration and $\mathrm{pH}$ of the reacting baths, temperature of deposition, and specific substrate treatment. In the present work, we have used SILAR technique to get nanoparticles of CdS. The SILAR deposition from aqueous solutions is a very promising method because of its simplicity and economy. The primary aim of the 
present work was to promote interest in SILAR method as applied to preparation of nanocrystalline cadmium sulphide powders. The deposition parameters were adjusted to give a nonadherent thin film which could be scratched to give powder. The structural and optical characterizations of the synthesized powders were carried out using X-ray powder diffractometer and UV-VIS spectrophotometer. Scanning electron microscopy was used to illustrate the formation of crystallites and transmission electron microscopy was used for particle size estimation.

\section{Materials and Methods}

Cadmium sulphide thin film was prepared by successive dipping of a precleaned glass substrate in cadmium acetate $\left[\mathrm{Cd}\left(\mathrm{CH}_{3} \mathrm{COO}\right)_{2} \cdot 2 \mathrm{H}_{2} \mathrm{O}\right]$ solution and ammonium sulphide $\left[\left(\mathrm{NH}_{4}\right)_{2} \mathrm{~S}\right]$ solution, both kept at room temperature. $0.1 \mathrm{M}$ cadmium acetate solution was used as cationic precursor. The anionic precursor was prepared by adding $5 \mathrm{cc}$ ammonium sulphide solution in $100 \mathrm{cc}$ distilled water. Dipping of the substrate in cationic precursors leads to the absorption of metal $\left(\mathrm{M}^{2+}\right)$ ions $\left[\mathrm{Cd}^{2+}\right.$ ion in this case] and subsequent dipping in anionic precursors leads to the absorption of sulpher $\left(\mathrm{S}^{2-}\right)$ ions. The reaction on the substrate leading to the formation of metal sulphide can be represented as

$$
\mathrm{Cd}^{2+}+\mathrm{S}^{2-} \longrightarrow \mathrm{CdS} \text {. }
$$

One complete set of dipping involves dipping of the substrate in cadmium acetate solution followed by dipping in anionic bath. Two hundred (200) such dipping was performed in order to get sufficient amount of powder. Between every immersion it is rinsed in ion exchanged water. Dipping of the substrate in cationic and anionic precursors leads to a very weakly adherent $\mathrm{CdS}$ film that could be easily scratched from the substrate. The powder obtained was thoroughly washed repeatedly in deionized water.

$\mathrm{X}$-ray diffraction (XRD) with $\mathrm{CuK}_{\alpha}$ radiation $(\lambda=$ $1.5418 \AA$ ) was made for structural characterization of the synthesized powders. The phase identification and crystalline properties of the films were studied by the X-ray diffraction (XRD) method employing a Philips PW 1830 X-ray diffractometer with $\mathrm{CuK}_{\alpha}$ radiation $(\lambda=1.5418 \AA)$. Utilizing the $\mathrm{X}$ ray diffraction data, the average crystallite size was estimated from Williamson-Hall equation $[15,16]$ :

$$
\beta \cos \theta=\frac{k \lambda}{D}+2 \varepsilon \sin \theta
$$

where $\lambda$ is the wave length of radiation used $\left(\mathrm{CuK}_{\alpha}\right.$ in this case), $k$ is the Scherrer constant, $\beta$ is the full width at half maximum (FWHM) intensity of the diffraction peak for which the particle size is to be calculated, $\theta$ is the diffraction angle of the concerned diffraction peak, $D$ is the crystallite dimension (or particle size), and $\varepsilon$ is the lattice strain. The slope of the plot of $\beta \cos \theta(y)$ against $2 \sin \theta(x)$ gives the strain.
In general the experimentally observed broadening $\left(\beta_{o}\right)$ is the total contribution from particle size and strain broadening $(\beta)$ and instrumental broadening $\left(\beta_{i}\right)$. Thus the broadening due to size and strain $(\beta)$ can be obtained from the experimentally observed broadening $\left(\beta_{o}\right)$ using the following relation:

$$
\beta=\beta_{o}-\beta_{i}
$$

The instrumental broadening arises from various factors such as nonparallelism of the incident X-ray beam and presence of other wavelengths apart from $\mathrm{CuK}_{\alpha}$ and it is a constant for a particular experimental setup. Diffraction data from standard silicon ( $\mathrm{Si}$ ) powder was used to measure the instrumental broadening [17]. The particle size is very high in the standard and the broadening in this case is due to instrument only. The broadening (in FWHM) against $2 \theta$ obtained from standard silicon sample was plotted in a graph and was used as reference. UV-VIS optical absorption measurement was performed in a spectrophotometer (Model UV-1800, Shimadzu, Japan) at room temperature. The spectra were recorded by using a similar glass as a reference. The band gap of the films has been calculated from the absorption edge of the spectrum.

\section{Results and Discussions}

Figure 1 shows the X-ray diffraction (XRD) pattern of CdS particles. The XRD was done after thorough washing of the synthesized powder in deionized water and heating the powder at $150^{\circ} \mathrm{C}$ for 2 hours. The material was scanned in the range $20-70^{\circ}$. The $2 \theta$ variation was employed with a 0.05 -degree step and a time step of 1 second. Intensity in arbitrary units is plotted against $2 \theta$ in Figure 1 . It is seen from the figure that peaks appear at $26.93^{\circ}, 44.33^{\circ}$, and $52.37^{\circ}$. The diffractogram of the sample reveals that all the peaks are in good agreement with the Joint committee on powder diffraction standard (JCPDS) data belonging to cubic CdS structure [18]. The corresponding reflecting planes are (111), (220), and (311), respectively.

$\mathrm{X}$-ray line broadening analysis to evaluate observed FWHM $\left(\beta_{o}\right)$ was carried out using computer software (MARQ2) [19]. The software utilizes Marquardt leastsquares procedure for minimizing the difference between the observed and simulated diffraction patterns. The peak-shape and intensity of reflection are modeled with a pseudo-Voigt $(\mathrm{pV})$ analytical function, which is a combination of a Gaussian function and a Lorentzian function. The background intensity is subtracted by fitting the background with a suitable linear function. From the values of $\beta_{o}$ obtained using MARQ2 fitting and the corresponding values of instrumental broadening (calculated from the standard $[20,21]$ ), $\beta$ was calculated using (3). Table 1 shows the values of $\beta_{o}, \beta_{i}$, and $\beta$. This value of $\beta$ was used to find the particle size using Scherrer equation [15] which, however, does not take account of strain broadening. The particle size was calculated using $k=0.9$, which corresponds to spherical crystallites, and $\lambda=$ $1.5418 \AA$, the wavelength of $\mathrm{CuK}_{\alpha}$ radiation. The average value 


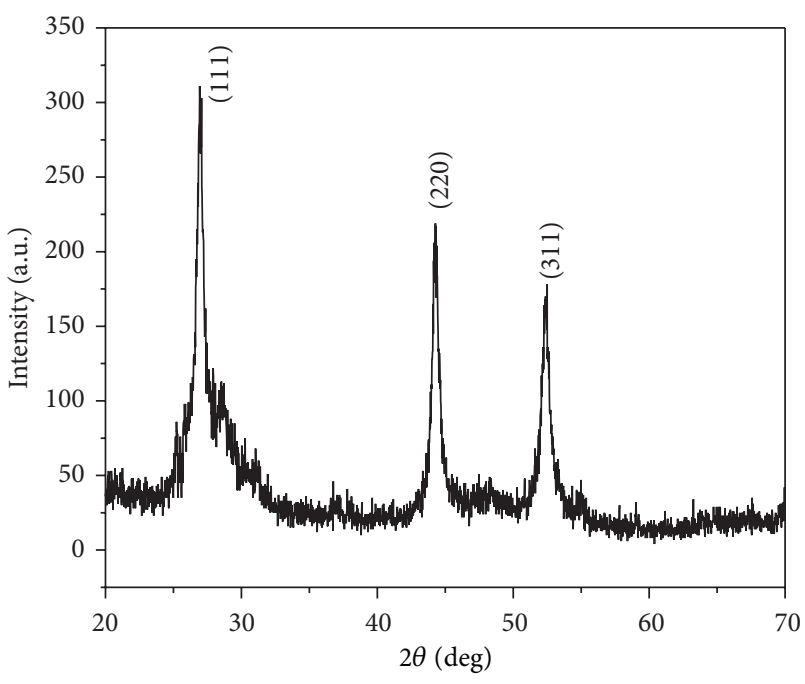

FIGURE 1: X-ray diffraction pattern of CdS nanoparticles.

TABLe 1

\begin{tabular}{lcccc}
\hline $\begin{array}{l}\text { Peak position } \\
(2 \theta)\end{array}$ & $\begin{array}{c}\beta_{o} \text { (in } \\
\text { degrees) }\end{array}$ & $\begin{array}{c}\beta_{i} \text { (in } \\
\text { degrees })\end{array}$ & $\begin{array}{c}\beta \text { (in } \\
\text { radians) }\end{array}$ & $\begin{array}{c}\text { Particle size } \\
(\AA)\end{array}$ \\
\hline 26.93 & 0.56 & 0.097 & 0.0081 & $\sim 177$ \\
44.33 & 0.62 & 0.100 & 0.0089 & $\sim 166$ \\
52.37 & 0.68 & 0.104 & 0.0101 & $\sim 153$ \\
\hline
\end{tabular}

of particle size taking instrumental broadening into account is $\sim 165.3 \AA$ (i.e., $\sim 16.5 \mathrm{~nm}$ ).

Figure 2 shows the plot of $\beta \cos \theta$ against $4 \sin \theta$ (Williamson-Hall plots or W-H plots). The slope of the plots represents average strain in the films whereas the inverse of intercept on $\beta \cos \theta$ axis gives the crystallite size $(D)$ according to (1). The average value of particle size was found to be $\sim 21.5 \mathrm{~nm}$ using $k=0.9, \lambda=1.5418 \AA$ and intercept on $y$-axis as 0.0065 . Thus the actual particle size taking strain into account is higher than that evaluated using Scherrer equation which does not take strain broadening into account. The estimated slope and hence value of lattice strain are $\sim 1.35 \times 10^{-3}$. The lattice strain introduced in the sample is possibly due to stacking fault within the crystallites. Since the deposition is carried out in ambient air, some stress is expected to be introduced which can result in stacking fault. The value of lattice strain is on the lower side compared to that reported $(0.0494)$ by chemical precipitation technique possibly due to high particle size of the samples [20]. The calculation of lattice strain was done by Williamson-Hall method. A value of RMS strain in the range $1.0 \times 10^{-3}$ to $3.0 \times 10^{-3}$ has been reported for $\sim 10 \mathrm{~nm}$ CdS powder synthesized by ball milling [22]. Lattice strain was calculated by Rietveld's method in their work.

SEM investigation (Figure 3) at normal incidence revealed formation of submicrometer crystallites. Figure 4 shows the TEM image of CdS. The particle size evaluated from TEM micrograph ranges approximately between 19 and $26 \mathrm{~nm}$ with an average of $\sim 23.5 \mathrm{~nm}$. The chemical

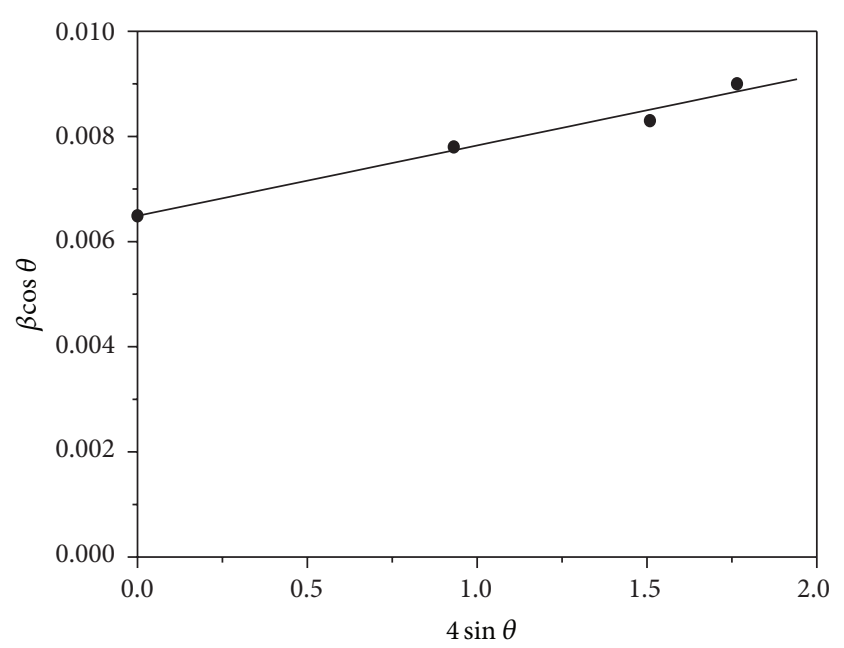

Figure 2: Williamson-Hall plot of CdS.

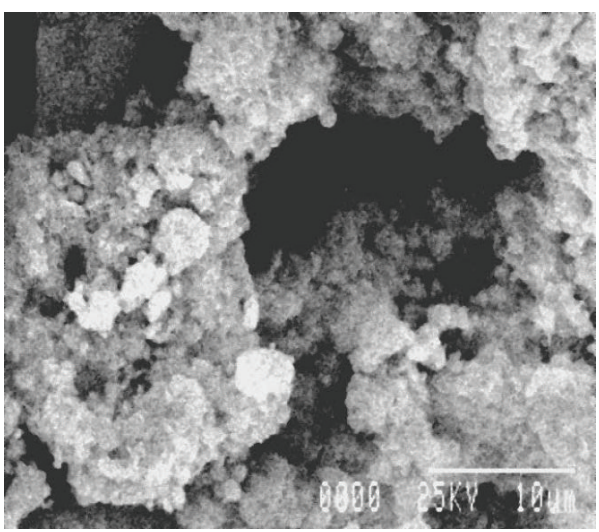

FIGURE 3: SEM image of CdS nanopowders.

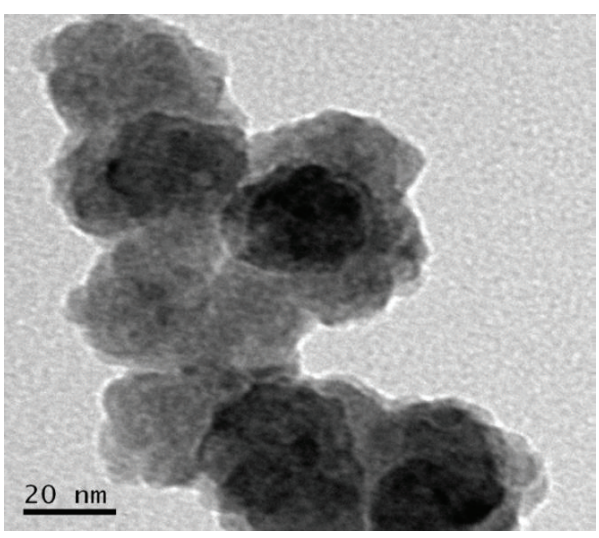

FIgURE 4: TEM image of CdS powder.

composition of the CdS was determined by EDX (energy dispersive X-ray) analysis (Figure 5), confirming the existence of $\mathrm{Cd}$ and $\mathrm{S}$ elements. The silicon and oxygen signal appears from the substrate. Contamination of trace amount of carbon and chlorine was detected in the films. The FTIR spectrum of CdS is shown in Figure 6. The absorption 


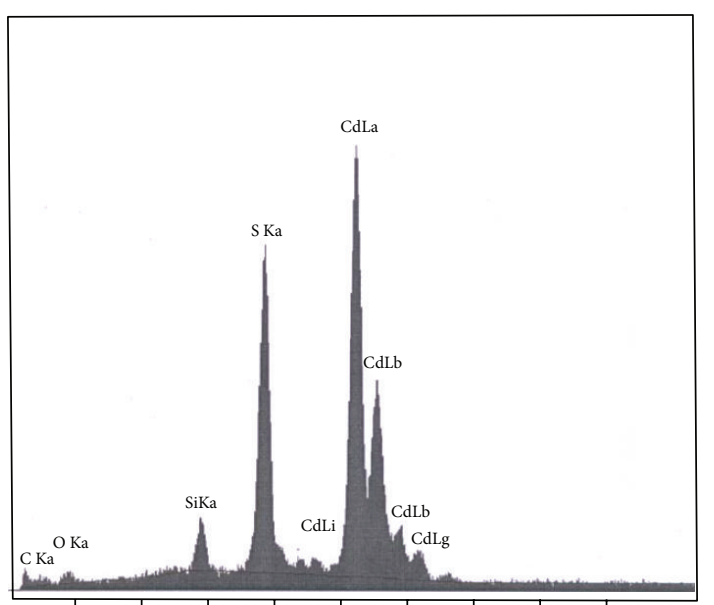

$\begin{array}{lllllllll}0.60 & 1.20 & 1.80 & 2.40 & 3.00 & 3.60 & 4.20 & 4.80 & 5.40\end{array}$ $(\mathrm{keV})$

Figure 5: EDX pattern of CdS.

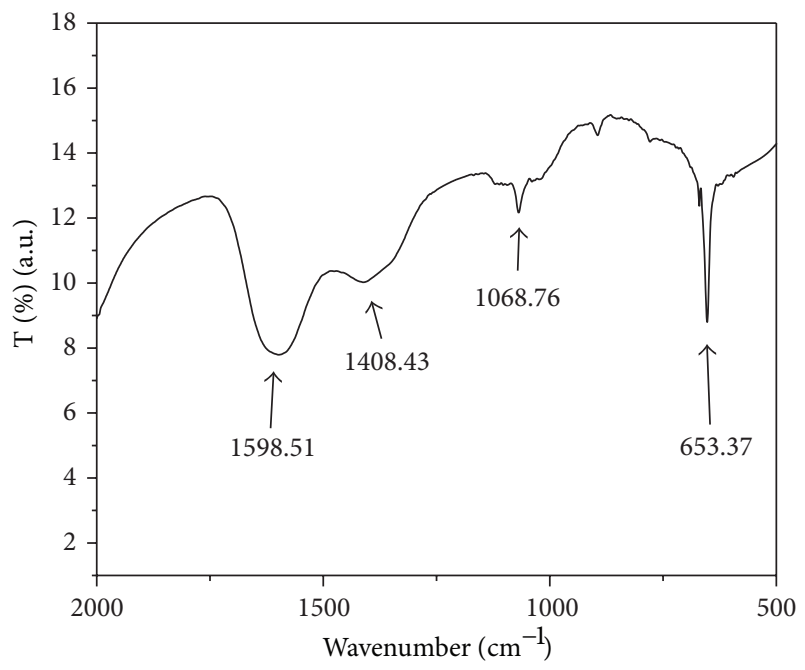

FIGURE 6: FTIR spectrum of CdS.

band observed at $653.37 \mathrm{~cm}^{-1}$ may be attributed to Cd-S stretching frequency $[23,24]$. The band at $1068.76 \mathrm{~cm}^{-1}$ may be attributed to $\mathrm{SO}_{4}{ }^{-}$traces and those at $1408.43 \mathrm{~cm}^{-1}$ and $1598.51 \mathrm{~cm}^{-1}$ may be attributed to sulphate group $(\mathrm{S}=\mathrm{O})$ and C-C stretching vibrations, respectively [23].

Absorption spectrum of CdS was recorded in the wavelength range $400-700 \mathrm{~nm}$. The optical absorbance (transmittance) depends on the density of point defects [21, 25]. Band gap energy $\left(E_{g}\right)$ was derived from the mathematical treatment of the data obtained from the absorbance versus wavelength with the following relationship:

$$
(\alpha h \nu)^{2}=A\left(h v-E_{g}\right)^{n / 2},
$$

where $v$ is the frequency, $\alpha$ is the absorption coefficient, $A$ is a function of index of refraction and hole/electron effective masses, $h$ is the Planck's constant, and $n$ carries the value of either 1 or 4 depending on whether the material has direct or

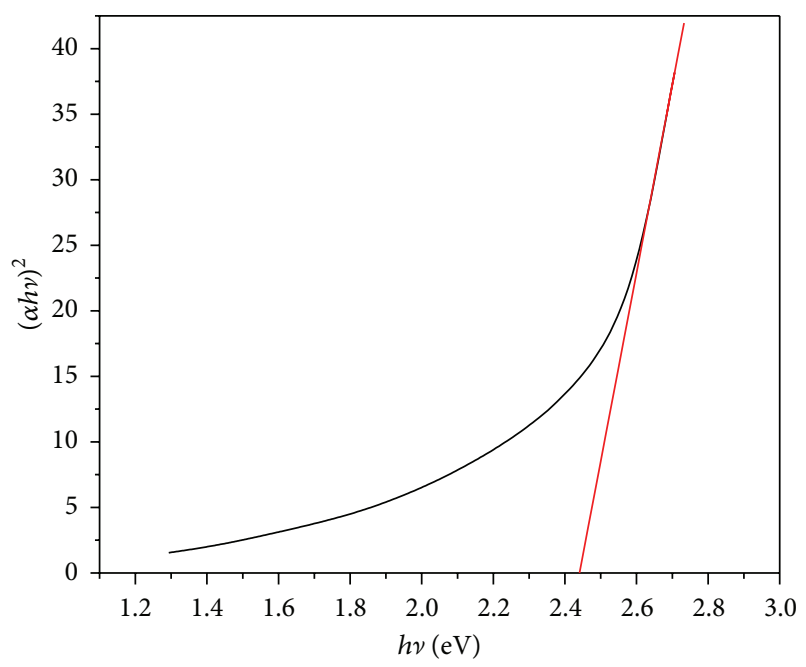

Figure 7: Photon energy (eV) dependence of $(\alpha h \nu)^{2}$.

indirect band gap. Since CdS has an indirect band gap, $n=4$ was used and a plot of $(\alpha h \nu)^{2}$ as a function of $h \nu$ was drawn (Figure 7). Extrapolation of the line to the base line, where the value of $(\alpha h \nu)^{2}$ is zero, gives $E_{g}$ as $2.44 \mathrm{eV}$.

The variation of resistance with temperature was studied in the temperature range. The sample for electrical measurement was prepared under high-pressure pellets. The electrical characterization was carried out by monitoring the variation of electrical resistance $(R)$ as a function of temperature $(T)$ using the conventional two probe technique with silver as electrodes. The film was kept in the dark inside a quartz tube furnace. Figure 8 shows the data on the variation of the electrical resistance with reciprocal temperature $(1000 / T)$ in the temperature range $325-425 \mathrm{~K}$. Electrical resistance was measured with a Keithley 6514 system electrometer. The conduction process may be described by the following equation:

$$
R=R_{o} \exp \left(\frac{E}{k T}\right) .
$$

In this equation, $R_{o}$ is the preexponential factor for the temperature range $300-400 \mathrm{~K}, k$ is Boltzmann's constant, $T$ is the absolute temperature, and $E$ is the activation energy for conduction. The experimentally obtained value of $E$ is $\sim 1.0 \mathrm{eV}$. An activation energy value of $0.9 \mathrm{eV}$ in the low temperature range has been attributed to grain boundarylimited conduction mechanism [26].

\section{Conclusions}

Nanocrystalline powders of CdS were prepared at room temperature and without use of any capping agent by SILAR technique. X-ray diffraction pattern indicates formation of cubic CdS. The CdS nanocrystalline powders formed have dimensions in the range $21-24 \mathrm{~nm}$ as evaluated by X-ray line broadening analysis and TEM measurements. Lattice strain evaluated by Hall-Williamson method was $\sim 1.35 \times$ $10^{-3}$. The characteristic stretching vibration mode of CdS at 


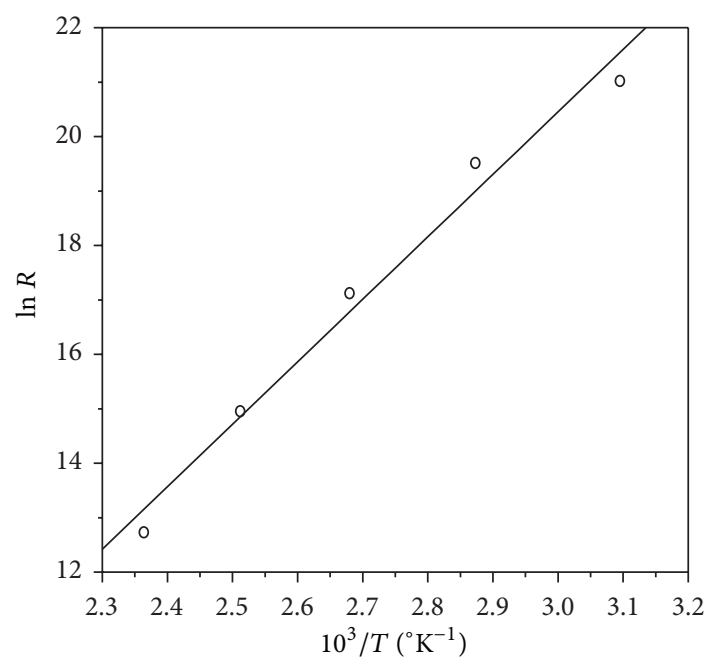

FIGURE 8: Temperature dependence of electrical resistance.

$653.37 \mathrm{~cm}^{-1}$ was observed in the absorption band in FTIR spectrum. Optical absorption study exhibits a band gap energy value of about $2.44 \mathrm{eV}$. The resistance of the material decreases with increasing temperature indicating semiconducting behavior. Temperature dependence of resistance follows an energy activation barrier value of $\sim 1.0 \mathrm{eV}$ which may be attributed to grain boundary-limited conduction mechanism. The technique of SILAR is simple and cost effective and offers an easily scalable alternative to industrial level. It seems that the technique can be extended for the preparation of other metal sulphide nanocrystals/quantum dots.

\section{Conflict of Interests}

The authors declare that there is no conflict of interests regarding the publication of this paper.

\section{Acknowledgment}

Authors wish to thank the University Grants Commission (UGC), India, for granting Centre for Advanced Study (CAS) under the thrust area "Condensed Matter Physics including Laser applications" to the Department of Physics, Burdwan University.

\section{References}

[1] D. Mandal, H. Hosoi, U. Chatterjee, and T. Tahara, "Direct observation of time-dependent photoluminescence spectral shift in CdS nanoparticles synthesized in polymer solutions," Journal of Chemical Physics, vol. 130, no. 3, Article ID 034902, 2009.

[2] R. Bhattacharya and S. Saha, "Growth of CdS nanoparticles by chemical method and its characterization," Pramana-Journal of Physics, vol. 71, no. 1, pp. 187-192, 2008.

[3] X. Peng, L. Manna, W. Yang et al., "Shape control of CdSe nanocrystals," Nature, vol. 404, no. 6773, pp. 59-61, 2000.
[4] R. R. Prabhu and M. Abdul Khadar, "Characterization of chemically synthesized CdS nanoparticles," Pramana-Journal of Physics, vol. 65, no. 5, pp. 801-807, 2005.

[5] N. Tessler, V. Medvedev, M. Kazes, S. Kan, and U. Banin, "Efficient near-infrared polymer nanocrystal light-emitting diodes," Science, vol. 295, no. 5559, pp. 1506-1508, 2002.

[6] J. Barman and K. C. Sarma, "Structural and optical properties of CdS nanoparticles," Indian Journal of Physics, vol. 82, no. 7, pp. 855-862, 2008.

[7] M. A. El-Sayed, "Small is different: shape-, size-, and composition-dependent properties of some colloidal semiconductor nanocrystals," Accounts of Chemical Research, vol. 37, no. 5, pp. 326-333, 2004.

[8] J. R. Lakowicz, I. Gryczynski, Z. Gryczynski, and C. J. Murphy, "Luminescence spectral properties of CdS nanoparticles," Journal of Physical Chemistry B, vol. 103, no. 36, pp. 7613-7620, 1999.

[9] J. R. Lakowicz, I. Gryczynski, Z. Gryczynski, K. Nowaczyk, and C. J. Murphy, "Time-resolved spectral observations of cadmium-enriched cadmium sulfide nanoparticles and the effects of DNA oligomer binding," Analytical Biochemistry, vol. 280, no. 1, pp. 128-136, 2000.

[10] V. Singh and P. Chauhan, "Synthesis and structural properties of wurtzite type CdS nanoparticles," Chalcogenide Letters, vol. 6, pp. 421-426, 2009.

[11] M. J. Pawar and S. S. Chaure, "Synthesis of cds nanoparticles using glucose as a capping agent," Chalcogenide Letters, vol. 6, no. 12, pp. 689-693, 2009.

[12] A. Dumbrava, C. Badea, G. Prodan, and V. Ciupina, "Synthesis and characterization of cadmium sulfide obtained at room temperature," Chalcogenide Letters, vol. 7, no. 2, pp. 111-118, 2010.

[13] T. Trindade, P. O’Brien, and X.-M. Zhang, "Synthesis of CdS and CdSe Nanocrystallites Using a Novel Single-Molecule Precursors Approach," Chemistry of Materials, vol. 9, no. 2, pp. 523-530, 1997.

[14] M. Sasagawa and Y. Nosaka, "Studies on the effects of Cd ion sources and chelating reagents on atomic layer Cds deposition by successive ionic layer adsorption and reaction (SILAR) method," Physical Chemistry Chemical Physics, vol. 3, no. 16, pp. 3371-3376, 2001.

[15] H. P. Klug and L. E. Alexander, X-Ray Diffraction Procedures for Polycrystalline and Amorphous Materials, Wiley, New York, NY, USA, 1974.

[16] B. D. Cullity and S. R. Stock, Elements of X-Ray Diffraction, Prentice Hall, Upper Saddle River, NJ, USA, 3rd ed edition, 2002.

[17] S. Patra, P. Mitra, and S. K. Pradhan, "Preparation of nanodimensional CdS by chemical dipping technique and their characterization," Materials Research, vol. 14, no. 1, pp. 17-20, 2011.

[18] "Joint Committee on Powder Diffraction Standards-JCPDS," Card no.10-0454, International Centre for Diffraction Data, Swarthmore, Pa, USA, 1995.

[19] S. Mondal and P. Mitra, "Preparation of Ni doped $\mathrm{ZnO}$ thin films by SILAR and their characterization," Indian Journal of Physics, vol. 87, no. 2, pp. 125-131, 2013.

[20] V. Singh and P. Chauhan, "Structural and optical characterization of CdS nanoparticles prepared by chemical precipitation method," Journal of Physics and Chemistry of Solids, vol. 70, no. 7, pp. 1074-1079, 2009.

[21] B. Saha, R. Thapa, S. Jana, and K. K. Chattopadhyay, "Optical and electrical properties of p-type transparent conducting 
$\mathrm{CuAlO} 2$ thin film synthesized by reactive radio frequency magnetron sputtering technique," Indian Journal of Physics, vol. 84, no. 10, pp. 1341-1346, 2010.

[22] S. Patra, B. Satpati, and S. K. Pradhan, "Quickest single-step mechanosynthesis of Cds quantum dots and their microstructure characterization," Journal of Nanoscience and Nanotechnology, vol. 11, no. 6, pp. 4771-4780, 2011.

[23] A. Sabah, S. A. Siddiqi, and S. Ali, "Fabrication and characterization of CdS nanoparticles annealed by using different radiations," World Academy of Science, Engineering and Technology, vol. 70, pp. 82-89, 2010.

[24] Y. Thangam and R. Anitha, "Surface sol-gel synthesis of cadmium sulfide fine particles in silica matrix," Journal of Research in Nanobiotechnology, vol. 1, no. 1, pp. 14-18, 2012.

[25] R. Thapa, B. Saha, S. Goswami, and K. K. Chattopadhyay, "Study of field emission and dielectric properties of AlN films prepared by DC sputtering technique at different substrate temperatures," Indian Journal of Physics, vol. 84, no. 10, pp. 1347-1354, 2010.

[26] P. P. Sahay, S. Jha, and M. Shamsuddin, "On the electrical conductivity of thermally evaporated CdS thin films," Journal of Materials Science Letters, vol. 21, no. 12, pp. 923-925, 2002. 

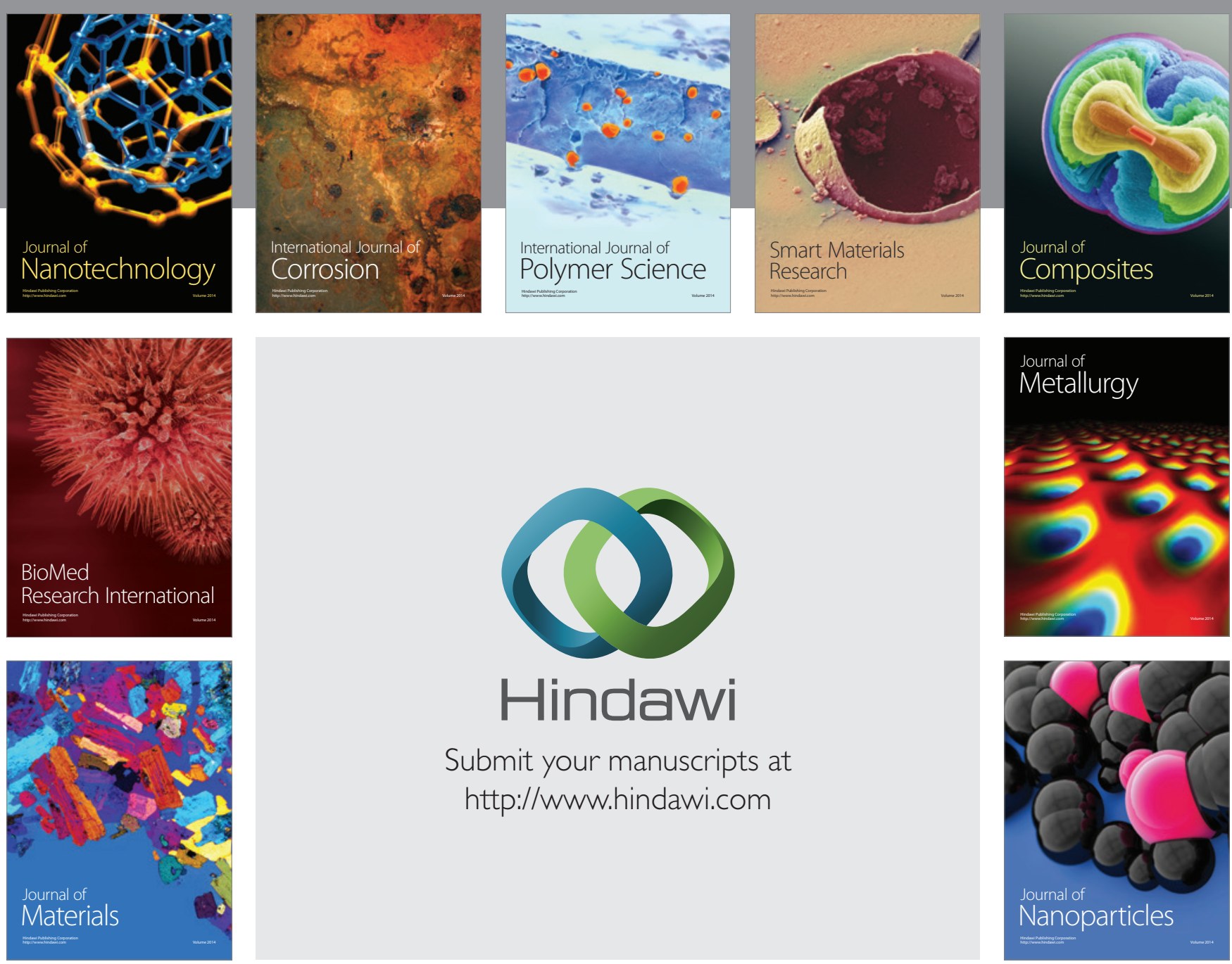

Submit your manuscripts at http://www.hindawi.com
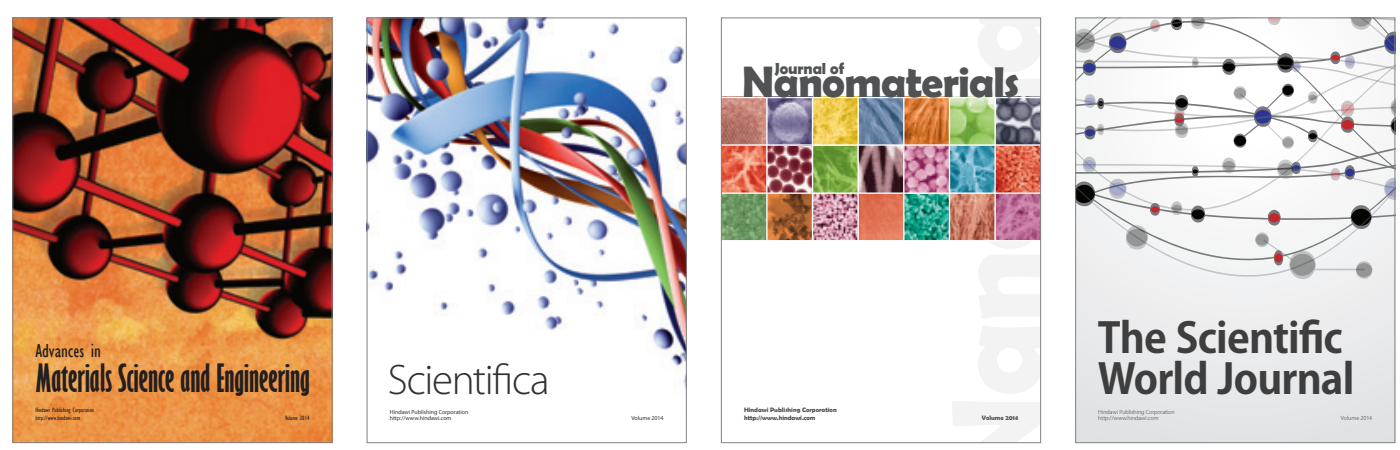

\section{The Scientific World Journal}
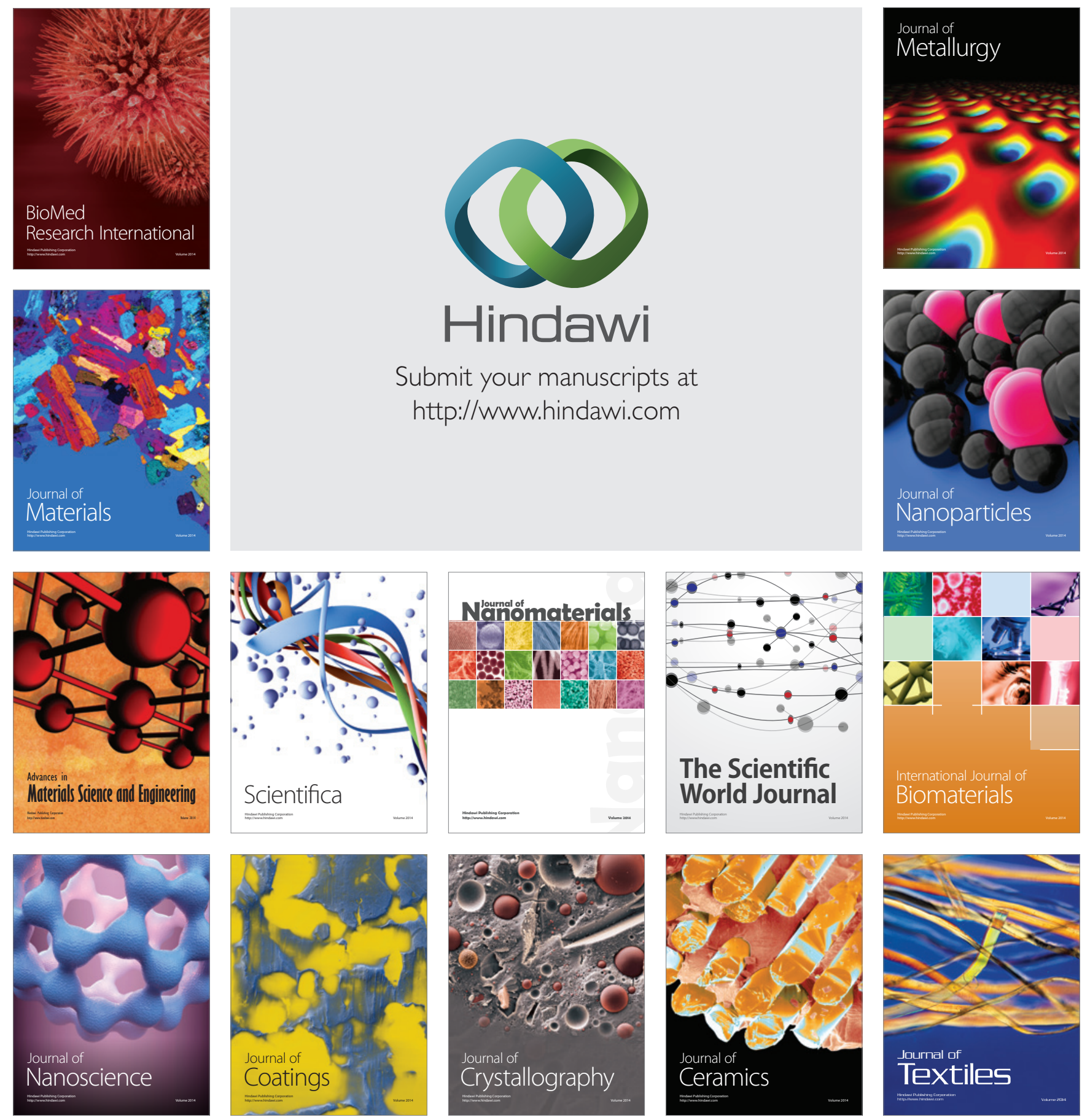